


\title{
TEST Y MEDIDAS EN FISIOTERAPIA PARA LAS DEFICIENCIAS CINÉTICAS EN NIÑOS CON PARÁLISIS CEREBRAL (PC)
}

\section{Test and Measures in Physical Therapy for the Kinetic Deficiencies in Children with cerebral palsy (PC)}

\author{
Fecha de Recepción: 15 de Mayo de 2012 - Fecha de Aprobación: 17 de Agosto de 2012
}

\author{
RESUMEN
}

Existen muchos estudios sobre parálisis cerebral acerca de fisiopatología incluyendo síntomas, signos y características de este trastorno, desafortunadamente el número de publicaciones realizadas en relación a la medición de deficiencias de orden cinético es escaso, profesionales como fisioterapeutas, que tienen como objeto de estudio el movimiento corporal humano, se ven limitados para cuantificar las mismas y por ende corroborar el resultado de las intervenciones. Por esta razón surge la necesidad de realizar una revisión sistemática, que arroje el panorama de test y medidas utilizadas para medir las deficiencias de orden funcional, en niños con parálisis cerebral (PC), para ello se buscan artículos en las bases de datos de Pubmed, Science Direct y Scielo, entre los años 2005 a 2012. El objetivo principal es reconocer cuales son los test y medidas usados en fisioterapia para la medición y evaluación de las deficiencias estructurales y funcionales que se presenten en niños con parálisis cerebral. De esta revisión se hallaron 1695 artículos de fisiopatología, etiología, características físicas, deficiencias motoras, sin embargo solo 19 abordan los diferentes test y medidas usados en fisioterapia para el manejo de niños con parálisis cerebral. En la literatura la antropometría y el desempeño muscular se miden de forma independiente, sin embargo, MACEDO y MILLER, contrastan sus teorías sobre características antropométricas y desempeño muscular de forma conjunta, frente a individuos con parálisis cerebral. Por último, la investigación concluye que la mayoría de evidencia encontrada analiza las deficiencias de integridad de nervios craneales y circulación.

PALABRAS CLAVE

\section{ABSTRACT}

Parálisis cerebral, test, medición, evaluación, fisioterapia.

Lots of studies talk about cerebral palsy andphysiopathology which includes symptoms, signs and characteristics; unfortunately, the number of publications in relation to the kinetic deficiency measurement is scarce. Professionals like physical therapists, whose their study aim is human corporal movement, are limited to quantify these publications and corroborate intervention results. For this reason, the necessity of making a systematic review that shows the panorama of tests and used measurements to evaluate and quantify functional deficiencies in children with cerebral palsy through articles search in data bases such as Pubmed, Science Direct and, Scielo between 2005 and 2012. The aim of this review is to recognize the tests and measurements that are used in Physical Therapy to assess and measure structural and functional deficiencies in children with cerebral palsy. In this part, 1695 articles were found about to physiopathology, etiology, physical characteristics and motor deficiencies; however, only 19 articles are related directly to our aim. Literature shows that anthropometry characteristics measurements and muscular performance are made independently, yet MACEDO and MILLER differ by measuring together these two characteristics in children with cerebral palsy. This investigation concludes that the majority of the evidence analyzes cranial nerves and circulation integrity deficiencies.

KEY WORDS

Cerebral Palsy, Test, Measures, Evaluation, Children, Physical Therapy. 


\section{INTRODUCCIÓN}

$\mathrm{L}$ a parálisis cerebral según Aparicio (1994) es un "trastorno persistente del movimiento y de la postura ocasionado por diversos procesos patológicos no progresivos que acontecen de un cerebro inmaduro".

Los diferentes estudios e investigaciones relacionados con el ámbito de la salud que hacen referencia al tema de parálisis cerebral (PC), contienen algunas perspectivas que abarcan causas, signos y síntomas que determinan el tipo y características generales de esta, pudiendo establecer los efectos visibles y no visibles a nivel anatomofisiologico, en cada uno de los sistemas, especialmente a nivel neurológico.

En concordancia con lo anterior, se destaca la parálisis cerebral como objeto de estudio, abordando el tema a nivel fisiopatológico, mostrando las deficiencias estructurales y funcionales desde una visión fisioterapéutica partiendo desde antecedentes de estudio, identificando un panorama de test y medidas utilizadas en el proceso evaluativo de los niños con Parálisis Cerebral, basados en la evidencia sobre mediciones específicas que definan dicha deficiencia con un claro enfoque terapéutico.

Según Nelson (2000), la parálisis cerebral a nivel epidemiológico, en Norteamérica afecta a 1,2- 2,5 de cada 1000 nacidos vivos, siendo moderada o grave, la de mayor prevalencia. Según O' Shea (2000), la evaluación de neurodesarrollo en los recién nacidos pretérminos, se debe hacer alrededor de los dos años de edad corregida, por esta razón se decide buscar literatura que incluyeran niños con rangos de edades de o a 6 años de edad.

Así mismo, la parálisis cerebral es una enfermedad de alto costo, según revisiones médicas, ingresos hospitalarios, repercusión en la productividad laboral, los costos en el año 2003 en Estados Unidos ascendían a 11500 millones de dólares.

Por esta razón es necesario un proceso acorde de examinación y evaluación de niños con parálisis cerebral para generar así un tratamiento que responda a las necesidades específicas de los individuos, por consiguiente una disminución de costos y tiempo tanto para los profesionales de la salud como para los niños.

Observando lo anteriormente expuesto, se genera la necesidad de determinar cuáles son los test y pruebas más indicados para ser aplicados, planteando un tratamiento estratégico, basado en los hallazgos analizados en la literatura.

Como consecuencia de lo anteriormente mencionado los fisioterapeutas se pueden ver limitados en su proceso de razonamiento clínico al no tener la evidencia necesaria durante el proceso de medición.

\section{METODOLOGÍA}

La búsqueda sistemática fue hecha a través de Pubmed, utilizando como palabras claves capacidad aeróbica, desempeño muscular, tono muscular, ventilación, respiración, intercambio, cognición, atención, parálisis cerebral, ortopedia, niños, fisioterapia, test, evaluación, medición y sus correspondientes relaciones. Se inició normalizando los términos en Dec's y posteriormente en MESH Database ubicada en Pubmed utilizando los conectores AND, OR, NOT; así mismo se delimito esta búsqueda por rangos de edad siendo de o a 6 años, entre los años de 2005 a 2012, en idioma inglés.

Los resultados de los artículos permitieron relacionar deficiencias como capacidad aeróbica, desempeño muscular, antropometría, estado de alerta, cognición y atención, aparatos asistidos y adaptativos, circulación, integridad de nervios craneales y periféricos, barreras ambientales de hogar y trabajo, ergonomía y mecánica corporal, desempeño muscular, integridad tegumentaria, movilidad articular, función motora, desarrollo motor e integración motora, postura y ventilación con un instrumento de medición.

Con base en las deficiencias mencionadas, se organizaron en una matriz, la cual incluía deficiencias, palabra de búsqueda, numero de artículos encontrados, utilizados y test hallados.

\section{RESULTADOS}

Se encontraron 1695 artículos en diferentes bases de datos, de los cuales se tomaron 19, que incluían test y medidas en fisioterapia para niños con parálisis cerebral. Para dar organización a la revisión, se tomó el modelo teórico que intenta desarrollar la temática, teniendo como base al patokinesis de la parálisis cerebral, las categorías de medición propuestas en la guía práctica de Fisioterapia descrita por la Asociación Americana de Fisioterapia (APTA, 2001), cabe aclarar que esta clasificación es basada en las deficiencias encontradas en el análisis fisiopatológico, estas son:

\section{Desempeño Muscular}

Es la "capacidad que posee el musculo o la fibra muscular para elongarse, así como, la velocidad de contracción y el balance".

Se hallaron un total de doscientos siete artículos de los cuales solamente cuatro reunian los criteros de inclusión, describiendo los siguientes test:

Se encontró la Electromiografía la cual se realizó específicamente de ocho músculos bilateralmente: trapecio (centro), erector de la columna (longuísimo), recto abdominal, oblicuo externo, glúteo mayor, glúteo menor, cuádriceps femoral (rectoanterior del muslo) y semitendinoso.

Asímismo se deben realizar movimientos específicos para generar las contracciones de músculos agonistas y antagonistas, dependiendo del movimiento, como inclinarse hacia atrás en posición sentada para activar los músculos rectos del abdomen, y levantar una pierna para activar los músculos contralaterales glúteo medio, dando como resultado una diferencia en la actividad muscular de tronco y cadera en las primeras etapas de la marcha en niños con parálisis cerebral en comparación con los niños con sin esta patología de base.

La media de frecuencia de esta, durante la marcha fue mayor y más variable de la zancada a zancada en todo el ciclo de la marcha 
para el grupo de PC de Desarrollo normal para los 8 músculos investigados.

La frecuencia media fue mayor en el grupo de niños con PC, lo cual sugiere la alteración de los patrones de activación muscular y reclutamiento de unidades motoras. Siendo resultado de mayores tasas de disparo a nivel del aumento en el número de unidades motoras reclutadas, o disminución de la sincronía.

Esto es consistente con la literatura, la cual sugiere una actividad muscular excesiva y descoordinada en PC. Al presentarse esto se contribuye la fatiga muscular en niños con parálisis cerebral.

Relacionándose con disminución de la eficiencia biomecánica, con cocontracción muscular, explicando una cantidad significativa de variables en el costo energético de la marcha, en niños con parálisis cerebral. El aumentó marcado paso a paso, los patrones de activación muscular en el grupo de PC puede implicar descoordinación y falta de madurez del comportamiento muscular, debido a un mayor cambio en las medidas de la marcha de otros, siendo una característica de patrones inmaduros.

En otro de los artículos se propone medir la fatiga muscular por medio de la bicicleta estacionaria para los niños con parálisis cerebral espástica dipléjica utilizando un diseño de ECA, en este articulo se propone el siguiente protocolo; Treinta sesiones de ejercicio se producirá en un período de 12 semanas.

La frecuencia óptima es de tres veces por semana durante un período de diez semanas, sin embargo, este esquema alternativo proporciona flexibilidad para las vacaciones, enfermedades $\mathrm{u}$ otros eventos. Cada sesión durará aproximadamente 60 minutos. Los sujetos se les pedirá que usar pantalones cortos y zapatillas de tenis durante cada sesión a efectos de comodidad, seguridad y evaluación. Ellos recibirán instrucción individualizada para un programa de ejercicio independiente de auto-estiramiento de los flexores de la cadera bilateral, extensor de la rodilla, los flexores de la rodilla y el tobillo músculos flexores plantares como a 5-10 minutos de calentamiento antes de la bicicleta. Otros grupos musculares que exhiben gama reducida de movimiento pueden ser incluidos en el programa de estiramiento para los sujetos individuales.

\section{Función Motora}

La función motora hace referencia a los patrones de movimiento que posee el individuo. Sin embargo en las personas que tienen parálisis cerebral se debe tener en cuenta que presentan un tono muscular alterado acompañado de reacciones asociadas, alteraciones a nivel del control motor selectivo y a nivel musculo esquelético, a su vez presenta alteraciones de control postural de equilibrio y fuerza.

Se encontraron cuatrocientos veintiocho artículos de los cuales dos reunian los criteros de inclusión y estos abarcaron los siguientes test:

La función de Gross Motor Sistema de Clasificación (GMFCS) se ha convertido en una herramienta importante para describir la función motora en los niños con Parálisis Cerebral (PC).
El Sistema de Clasificación de Habilidad Manual (MACS) se desarrolló recientemente, en una clasificación correspondiente de esta, por medio de un programa de fisioterapia y terapia ocupacional, el cual dio como resultado que la GMFCS y MACS son a menudo discrepantes en individuos con Paralisis Cerebral otorgando un cuadro clínico completo y arrojando las necesidades específicas de cada individuo.

\section{Antropometría}

Es la medición de la composición del cuerpo reflejada en el Índice de Masa Corporal, acompañado de la medida de pliegues cutáneos , altura y peso entre otros, en el caso de los individuos con parálisis cerebral se debe tener en cuenta que ellos poseen, un patrón de crecimiento distinto al de los niños normales debido a que tienen distinta composición corporal, con disminución de la densidad ósea, la masa muscular, la masa grasa y el crecimiento lineal y patrones de desarrollo puberal y de edad ósea diferentes."

Se localizaron un total de cincuenta y cuatro artículos de los cuales solamente tres reunían los criterios de inclusión estipulados.

En la revisión sistemática se encontraron aplicaciones antropométricas como método de examinación en individuos con Paralisis Cerebral por medio de la toma de medidas de pliegues cutáneos, sin embargo es prioritario obtener como dato primario el peso corporal del individuo el cual según la evidencia fue medido con la báscula Welmy digital balance (model RIW 200) y las unidades de medida fueron Kilogramos, posteriormente se definieron las regiones en las cuales se realizarían las mediciones, el espesor del tejido subcutáneo se midió tres veces en cada región para las mitades dominantes y no dominante del cuerpo.

Estos fueron utilizados posteriormente como la media calculada para cada pliegue de la piel.

Después de calcular la media para uno, se hicieron comparaciones dentro y entre los grupos, teniendo como unidad de medida, milímetros.

En el marco de las observaciones anteriores cabe aclarar que los datos obtenidos del hemicuerpo dominante con respecto al contralateral se ven condicionadas por el tipo de Parálisis Cerebral y limitaciones físicas así mismo la manera en la cual desempeña sus actividades básicas cotidianas.

Las alteraciones neuromusculares de la parálisis cerebral, tales como contracciones musculares y movimientos involuntarias hacen que sea difícil medir con precisión su composición corporal durante la evaluación fisioterapéutica, como consecuencia la mayoría de la población con dicho trastorno, nopuede ser evaluado desde el punto de vista nutricional, utilizando los métodos convencionales tales como la altura y la masa corporal, porque muchos de estos niños presentan retraso del crecimiento o asimetría en su composición corporal.

En vista de esto, el uso de tablas de correlación de peso y la altura no es suficiente para estimar el Índice de Masa Corporal y el porcen- 
2 taje de tejido adiposo, es por tal motivo la importancia del uso de la antropometría como herramienta de medición, la cual arroja valores cuantificables, que le permite al profesional de la salud realizar un seguimiento del proceso de crecimiento del individuo.

\section{Capacidad Aeróbica}

Se define como la "Capacidad del cuerpo de producir energía en forma permanente y prolongada a partir de diversos nutrientes, siendo este un suministro de oxígeno, es la posibilidad de procesar este intracelularmente y así producir la suficiente cantidad de energía durante exigencias físicas, vigorosas y de larga duración, se puede expresar como mililitros de oxígeno por minuto y por kilogramo de peso corporal".

En este ítem se encontraron un total de treinta y ocho artículos de los cuales solamente dos reunían los criterios de inclusión estipulados, en estos se resalta el uso de la bicicleta estática como herramienta a nivel físico con el fin de mejorar la fuerza y la capacidad cardiorrespiratoria.

Sin embargo lo anteriormente mencionado está condicionado por la intensidad de las sesiones la frecuencia y la duración de cada sesión.

Para cada sujeto se debe manejar una resistencia acorde a sus capacidades motoras aumentando progresivamente la misma. Se inicia la sesión con intervalos de un minuto de ejercicio y dos minutos de recuperación o descanso, logrando así aumentar la capacidad cardiorespiratorio.

La intervención de ciclismo se divide en dos fases: 1) Fortalecimiento de la extremidad inferior y 2) La resistencia cardiorrespiratoria.

\section{Estado de Alerta, Cognición y Atención}

Según la guía práctica de Fisioterapia descrita por la Asociación Americana de Fisioterapia (APTA) "Evalúa las funciones mentales superiores que le permiten al paciente desarrollarse de una manera autónoma y segura frente a su estado"

En esta categoría se hallaron un total de ciento veintisiete artículos de los cuales solamente cinco reunian los criteros de inclusión.

Como resultado se evidencio que el análisis factorial confirmatorio apoyó unidimensionalidad escala, la dependencia de elemento local, y la invariancia. Las puntuaciones de las simulaciones por ordenador de los CAT prototipos con diferentes reglas de detención fueron consistentes con las cuentas del banco completo del artículo $(\mathrm{r}=.93 \mathrm{a} .98)$. Las puntuaciones de resumen de actividades discriminadas en todos los niveles de las extremidades superiores y la gravedad de motricidad gruesa, se correlacionaron con el instrumento de recolección de datos de resultados de Pediatría (PODCI) la función física y el deporte subescala $(\mathrm{r}=0,86)$, la Medida de Independencia Funcional de la Infancia (Wee-FIM) $(\mathrm{r}=0,79)$, y la versión pediátrica de calidad de vida de Parálisis Cerebral-Inventario $(\mathrm{r}=0,74)$.
A continuación (Tabla 1) se muestra los resultados de la revisión sistemática clasificada por variables, la cantidad de artículos encontrados en cada una de ellas y finalmente los artículos que cumplen los requisitos determinados en los criterios de inclusión explicados anteriormente.

\begin{tabular}{|c|c|c|c|c|}
\hline CATEGORIA & $\begin{array}{c}\text { PALABRAS } \\
\text { DE BUSQUEDA }\end{array}$ & 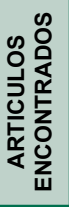 & 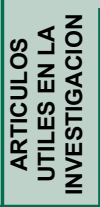 & $\begin{array}{l}\text { TEST Y MEDIDAS } \\
\text { ENCONTRADOS }\end{array}$ \\
\hline \multirow{2}{*}{$\begin{array}{l}\text { Capacidad } \\
\text { Aeróbica }\end{array}$} & $\begin{array}{l}\text { Tests to Assess } \\
\text { Aerobic Capacity in } \\
\text { Cerebral Palsy }\end{array}$ & 17 & 2 & \multirow[t]{2}{*}{ Bicicleta Estática } \\
\hline & $\begin{array}{l}\text { Aerobic Capacity Tests } \\
\text { in Cerebral Palsy }\end{array}$ & 21 & 0 & \\
\hline $\begin{array}{c}\text { Categorías } \\
\text { Antropométricas }\end{array}$ & $\begin{array}{l}\text { Fat Percentage Test in } \\
\text { Cerebral Palsy }\end{array}$ & 43 & 3 & $\begin{array}{l}\text { Densitometría } \\
\text { Absorciometria } \\
\text { Resonancia Magnética }\end{array}$ \\
\hline \multirow[b]{2}{*}{$\begin{array}{l}\text { Estado de Alerta, } \\
\text { Cognición y } \\
\text { Atención }\end{array}$} & $\begin{array}{l}\text { Measuring the } \\
\text { Percentage of Body Fat } \\
\text { in Cerebral Palsy }\end{array}$ & 11 & 0 & $\begin{array}{l}\text { Ecuaciones Basadas } \\
\text { en Musculo Tríceps y } \\
\text { Subescapular. }\end{array}$ \\
\hline & $\begin{array}{l}\text { Test of Cognition } \\
\text { for Children with } \\
\text { Cerebral Palsy }\end{array}$ & 127 & 5 & $\begin{array}{l}\text { CAT (Pruebas } \\
\text { computarizadas de } \\
\text { Adaptación) Escala de } \\
\text { Conners GMFCS MACS }\end{array}$ \\
\hline $\begin{array}{l}\text { Integridad de } \\
\text { Nervios } \\
\text { Craneales } \\
\text { y Periféricos }\end{array}$ & $\begin{array}{l}\text { Integrity Assessment } \\
\text { of Cranial Nerve in } \\
\text { Cerebral Palsy }\end{array}$ & 11 & 0 & $\begin{array}{l}\text { No se encontró ningún } \\
\text { tipo de test. }\end{array}$ \\
\hline \multirow{2}{*}{$\begin{array}{l}\text { Barreras } \\
\text { Ambientales } \\
\text { de Hogar } \\
\text { y Trabajo }\end{array}$} & $\begin{array}{l}\text { Tests to Asses } \\
\text { Environmental Barriers } \\
\text { of Children with Cerebral } \\
\text { Palsy }\end{array}$ & 15 & 1 & \multirow{2}{*}{$\begin{array}{l}\text { Encuesta FPQ } \\
\text { (Encuesta de } \\
\text { Frecuencia de } \\
\text { Participación) }\end{array}$} \\
\hline & $\begin{array}{l}\text { Tests to Asses } \\
\text { Environmental Barriers } \\
\text { of Children with Cerebral } \\
\text { Palsy }\end{array}$ & 17 & 0 & \\
\hline \multirow{2}{*}{$\begin{array}{l}\text { Ergonomía } \\
\text { y Mecánica } \\
\text { Corporal }\end{array}$} & $\begin{array}{l}\text { Evaluation of } \\
\text { Ergonomics in Cerebral } \\
\text { Palsy }\end{array}$ & 24 & 0 & \multirow{2}{*}{$\begin{array}{l}\text { No se encontró ningún } \\
\text { tipo de test. }\end{array}$} \\
\hline & $\begin{array}{l}\text { Tests to Assess Body } \\
\text { Mechanics in Cerebral } \\
\text { Palsy }\end{array}$ & 12 & 0 & \\
\hline $\begin{array}{l}\text { Desempeño } \\
\text { Muscular }\end{array}$ & $\begin{array}{l}\text { Evaluation of Muscle } \\
\text { Performance in Cerebral } \\
\text { Palsy. }\end{array}$ & 207 & 5 & $\begin{array}{l}\text { Electromiografía } \\
\text { Bicicleta Estática con } \\
\text { Inclinación } \\
\text { Escala de Tardieu } \\
\text { Fatiga Muscular (Mayor } \\
\text { cantidad de repeticiones } \\
\text { en un segmento corporal. }\end{array}$ \\
\hline Tegumentario & $\begin{array}{l}\text { Skin Tests to Evaluate } \\
\text { the Cerebral Palsy }\end{array}$ & 1 & 0 & $\begin{array}{l}\text { No se encontró ningún } \\
\text { tipo de test. }\end{array}$ \\
\hline Circulacion & $\begin{array}{l}\text { Test to Assess the } \\
\text { Circulation in Cerebral } \\
\text { Palsy }\end{array}$ & 159 & 0 & $\begin{array}{l}\text { No se encontró ningún } \\
\text { tipo de test. }\end{array}$ \\
\hline
\end{tabular}

Tabla 1: Resultados Revisión Sistemática en las Bases de Datos Pubmed, Scielo, especificando palabras claves, los años abarcados y numero de artículos encontrados.

\section{DISCUSIÓN}

Como lo han evidenciado varios autores como MACEDO y MILLER, los individuos que presentan Paralisis Cerebral al evaluar su 
composición corporal, la observación no es suficiente, ya que todos los niños presentan diferentes características antropométricas, por tal motivo los métodos que usan los fisioterapeutas deben modificarse y ser complementados.

La antropometría es uno de los métodos más accesibles y económicos a los cuales se tiene acceso al momento de evaluar, sin embargo este se puede complementar con la resonancia magnética y ecuaciones que brinden resultados o confirmación de estos acerca de acumulación de tejido adiposo, su porcentaje y zonas específicas de mayor prevalencia.

Así mismo se debe tener en cuenta que todos los resultados están condicionados por factores externos como los niveles de desnutrición, tipo de Paralisis cerebral, nivel de funcionalidad y desempeño de actividades en la vida diaria.

Lo anteriormente mencionado tiene directa relación con el desempeño a nivel muscular, ya que al presentarse un mayor porcentaje de tejido graso y poca masa muscular se presenta fatiga muscular más rápido ante estímulos de carga externa.

Al realizar estudios de la activación de músculos específicos, se ha encontrado que la actividad muscular del tronco y la cadera en las primeras etapas de la marcha, en niños con parálisis cerebral, en comparación con los niños sin este trastorno de base, tienen menor frecuencia en la activación muscular durante el proceso de marcha y más variable de la zancada a zancada en todo el ciclo de la marcha para el grupo de PC de Desarrollo normal.

Lo cual sugiere la alteración de los patrones de activación muscular y reclutamiento de unidades motoras. Esto puede ser resultado de mayores tasas de disparo a nivel nervioso lo cual genera un aumento del número de unidades motoras reclutadas, o disminución de la sincronía de las unidades motoras. Esto es consistente con la literatura ya que sugiere que la actividad muscular excesiva y descoordinada en PC contribuye a la fatiga muscular en niños con parálisis cerebral al realizar actividades de la vida cotidiana.

Según lo anteriormente expuesto se guarda una relación directa a lesiones neurológicas dadas por los factores causantes de la Paralisis Cerebral, los cuales generan alteraciones a nivel cognitivo dificultando la respuesta ante estímulos al realizar actividades, funciones del habla , funciones de compresión intelectual, entro otros.

Como se logró observar a lo largo de este escrito la Paralisis Cerebral no solo afecta la parte sistémica y de funciones corporales, sino que esta a su vez limita el desarrollo del individuo en todos sus ámbitos, para lograr comprender todo el proceso es necesario entender el proceso fisiopatológico dados desde el momento de la gestación o en el momento del parto a presentarse la asfixia prenatal una de las causas de mayor prevalencia de la Paralisis Cerebral.

$\mathrm{Al}$ abordar este proceso se debe tener claro los tipos y niveles de severidad de las lesiones cerebrales y como estos, afectan los diferentes sistemas para lograr así un tratamiento dirigido específicamente a las necesidades propias de cada individuo.

Por tal razón, se deben conocer los diferentes test y medidas utilizados en esta población, sin embargo al realizar la búsqueda de evidencia es notorio que este campo no ha sido explorado a profundi$\mathrm{dad}$, por lo cual es necesario generar nuevo conocimiento logrando así test y medidas que puedan aplicar el personal de salud específicamente los fisioterapeutas ya que su rol en la rehabilitación y la recuperación del mayor grado de funcionalidad es vital en el desarrollo y evolución del individuo. 


\section{Referencia:}

\section{Las referencias a otras obras son una parte muy importante en la literatura científica; ya que estas permiten conocer más sobre los autores y mantener vivas sus voces dentro del texto.}

1. Amezcua. (1996). Evaluación de Programas Sociales. Edición DíazMa T. Fernández López, A. Pelegrín Molina; Necesidades educativas especiales del alumnado con discapacidad física.

2. Aspudillo.J. Evaluación de la unidad feto-placentaria. Chile

3. Bonilla F, Pellicer A. (2008). Obstetricia, reproducción Y ginecología básicas; Ed. Panamericana.

4. Borges.J (1998). Examen neurológico. (Copyright) p.p 100-188Stokes. (2007) .Fisioterapia en la Rehabilitación Neurológica. Elsevier Mosby

5. Cabero, L. (2007). Obstetricia y Medicina Materno - Fetal. Editorial Medico Panamericana.

6. Castellanos, R. Gerardo R. (2010). Asociación entre factores Perinatales y neonatales de Riesgo y parálisis cerebral. Rev. Cubana Pediatr, vol.82, n.2, pp o-o. ISSN $0034-7531$.

7. Ceriani, C. F, Mariani, J, Lupo. (2009). Neonatología Practica, Cuarta Edición. Editorial Médica Panamericana.

8. Curso de "Máster en Igualdad y Género en el ámbito público y privado" Interuniversitario-Internacional 2007/2009. URL: http://chiariargentina.jimdo.com/sistema-nervioso-central/

9. Delgado, M. (1994).Fundamentación anatómico funcional del rendimiento y del entrenamiento de la resistencia del niño y del adolescente. Rev Motricidad. 1, 95-108

10. Duque. F. (2003). Crestas neurales, placodas y arcos branquiales: una revisión evolutiva y embriológica de datos básicos y recientes.

11. Eileen Fowler G, M Knutson L, Sharon K DeMuth, Sugi M, Siebert, Kara, Simms V, Stanley P A, Winstein C (2007); Pediatric endurance and limb strengthening for children with cerebral palsy (PEDALS) - a randomized controlled trial protocol for a stationary cycling intervention; BMC Pediatrics , $7: 14$

12. Fejerman, N. (2007). Neurología Pediátrica. Editorial Médica Panamericana. ISBN:84-7978-184-X, pág. 1046.

13. Gonzalez, G. (2012). Comunicación y Lenguaje en la Discapacidad Motorica. Archivo en Línea consultado el 4 de Enero.

14. Guyton, H. (2007) Tratado de Fisiología Medica, Décima Edición. Editorial Mc Graw Hill

15. Hûter A., Schewe H., Heipertz W. (2006) Fisiología y teoría del entrenamiento, Ed. Paidotribo.
16. Jacobs. Introducción a la Investigación Pedagógica, (Medición - Requisitos - Procedimientos para construir un Instrumento de Medición) Introducción a la Investigación Pedagógica de Ary, Jacobs y Razavieh

17. Johnson D, Freeman M, Pravesh S, Modlesky C. (2008). .The Journal of Pediatrics 01 de mayo 2009 (volumen 154 número 5 Páginas 715-720. e1

18. Mac K, Mackenzie ICK, Polani PE. (1959). The Little Club: Memorandum on terminology and classification of — cerebral palsy $\square$. Cerebral Palsy Bulletin.

19. Macedo, Osmair Gomes de et al .Comparative study of skin folding of dominant and nondominant hemibodies in spastic hemiplegic cerebral palsy. Clinics, $\hat{A}$ SÃEo Paulo, Â v. 63, Â n. 5, ÂÂA 2008 . Â Available from <http://www.scielo.br/scielo.php

20. Martínez. E. Capacidad aeróbica. Medellín.

21. Malagon, J. (2007). Parálisis Cerebral. Clínica para la Atención del Neurodesarrollo, México Medicina.

22. Mellado.P, Sandoval. P (2002). Encefalopatía hipóxico - isquémica. Universidad Católica de Chile. Vol. XXVI. URL:http://lahondurasvaliente.blogspot.com/2011_11_01_archive.html

23 Mellado.P, Sandoval. P. (2002). Encefalopatía hipóxico-isquémica. Universidad Católica de Chile. Vol. XXVI.

24. Morilla. B, Morales. M, Ruiz. Barranco. Hipoxia perinatal.

25. Muzzo.S.(2003). Normal and abnormal growth of the child and the adolescent.Unidad de Endocrinología.

26. Noelle G. Moreau, Li, Geaghan J, Damián D, (2008).Resistencia a la fatiga durante una tarea de cumplimiento voluntario se asocia con menores niveles de movilidad en la parálisis cerebral, Archivos de Medicina Física y Rehabilitación, Volumen 89, Número 10, páginas 20112016, ISSN 0003-9993, 10.1016/j.apmr.2008.03.012.

27. Olavarria. A. Biomecánica y flujometria Doppler de la Unidad Úteroplacento-fetal.

28. Ogas. M (2006). Síndrome de aspiración de líquido amniótico meconial. Hospital Universitario de Maternidad y neonatología. (p.p 1-2) Córdoba. 
